\title{
Three-Dimensional Plasmonic Nanostructure Design for Boosting Photoelectrochemical Activity
}

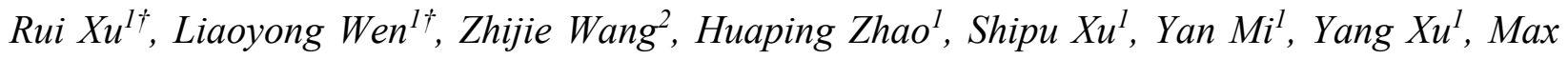
Sommerfeld ${ }^{l}$, Yaoguo Fang ${ }^{l}$, Yong Lei ${ }^{I^{*}}$

${ }^{l}$ Institute for Physics \& IMN MacroNano (ZIK), Ilmenau University of Technology, Unterpoerlitzer Straße 38, 98693 Ilmenau, Germany and ${ }^{2}$ Key Laboratory of Semiconductor Materials Science, Institute of Semiconductors, Chinese Academy of Sciences, Beijing 100083, PR China.

KEYWORDS: photonic mode, surface plasmon resonance, three-dimensional nanostructure, photoelectrochemical, $\mathrm{CdS} / \mathrm{Au}$

\begin{abstract}
Plasmonic nanostructures have been widely incorporated into different semiconductor materials to improve solar energy conversion. An important point is how to manipulate the incident light so that more light can be efficiently scattered and absorbed within the semiconductors. Here, by using a tunable three-dimensional Au pillar/truncated-pyramid (PTP) array as a plasmonic coupler, a superior optical absorption of about $95 \%$ within a wide wavelength range is
\end{abstract}


demonstrated from an assembled CdS/Au PTP photoanode. Based on incident photon to current efficiency measurements and the corresponding FDTD simulations, it is concluded that the enhancement is mainly attributed to an appropriate spectral complementation between surface plasmon resonance (SPR) modes and photonic modes in the Au PTP structure over the operational spectrum. Because both of them are wavelength dependent, the Au PTP profile and CdS thickness are further adjusted to take full advantages of the complementary effect and subsequently, an angle independent photocurrent with an enhancement of about $400 \%$ was obtained. The designed plasmonic PTP nanostructure of $\mathrm{Au}$ is highly robust and it could be easily extended to other plasmonic metals equipped with semiconductor thin film for photovoltaic and photoelectrochemical cells.

Solar energy conversion is a significant approach to providing a sustainable and fossil-free energy source. ${ }^{1-5}$ Photoelectrochemical (PEC) water splitting, which could convert solar energy into chemical energy, has been intensively investigated over the last few decades. ${ }^{6-14}$ One of the main challenges that limit the conversion efficiency of PEC electrodes is the severe trade-off between the long penetration depth of photons and the relatively short distance of carrier extraction at the electrode/electrolyte interface. ${ }^{15,16}$ Incorporating plasmonic nanostructures into a thin semiconductor is a promising route to address this issue, because surface plasmon resonance (SPR) that originates from the plasmonic nanostructures can give rise to intriguing phenomena, including guiding electromagnetic (EM) radiation and enhancing near-filed electric field etc. ${ }^{17-24}$ By properly constructing plasmonic nanostructure, the incident light could be folded into the active material through directional scattering and intense-electric-field aided light 
absorption around the plamonic nanostructures to achieve a film of electrical thinness and optical thickness. ${ }^{25-27}$ Some attempts, like embedding gold nanoparticles into a thin hematite $\mathrm{Fe}_{2} \mathrm{O}_{3}$ film, ${ }^{28}$ or integrating gold pillars within CdS nanorod arrays, ${ }^{29}$ were tried to incorporate these SPR features into PEC cells. However, SPR modes are typically suppressed in the short wavelength range (e.g., $<500 \mathrm{~nm}$ for gold because of the strong intrinsic interband light absorption). ${ }^{17}$ In order to achieve a better light utlization, solar spectrum with short wavelengths needs to be concerned as well. Photonic modes that are capable of reducing reflection and inducing diffraction effect to the short wavelength range have been widely adopted, such as nanoengineering semconductor films into archetectures with gradient refractive index profiles or manufacturing subwavelength periodic stuctures. ${ }^{30-36}$ Moreover, SPR and photonic modes can work in synergy and compensate each other over a wide wavelength range. Such as, by conformally coating gold nanoparticle arrays with a thin hematite $\mathrm{Fe}_{2} \mathrm{O}_{3}$ film, an improved light utilization has been reported, in which the contributions of the photonic modes start over $500 \mathrm{~nm}$ and the SPR modes dominate at around $650 \mathrm{~nm} \cdot{ }^{37}$ However, the zero dimensional nanostructure only supports SPR modes at discrete wavelengths, which largely limits SPR function domain. ${ }^{38-}$ ${ }^{41}$ Meanwhile, the zero dimensional surface profile leads to a poor tunability of the photonic modes. Thus, in order to broaden the SPR optical region and photonic mode induced light trapping region, as well as to maximize their complementary absorption range, competent design of nanostructure architecture is highly requested.

In this work, an approach was proposed to construct a three-dimensional (3D) array of pillar/truncated-pyramid (PTP) Au plasmonic nanostructure on a large scale of centimeter-sized range, in which each unit is composed of a pillar (P) setting on a truncated-pyramid (TP). To demonstrate the advantages of the Au PTPs for light absorption, a uniform semiconductor (e.g., 
CdS) film was deposited to form a CdS/Au PTP photoanode. Comparing to CdS/Au plane and pillars, a continuous incident photon to current efficiency (IPCE) enhancement from 300 to 600 nm was observed for the CdS/Au PTPs. Along with detailed FDTD simulation, we concluded that the IPCE enhancement above $450 \mathrm{~nm}$ is mainly originated in SPR modes, whereas the IPCE enhancement below $450 \mathrm{~nm}$ is dominated by the photonic modes. Systematic studies on the aspect ratio of $\mathrm{P} / \mathrm{TP}$ and the thickness of CdS film were carried out to reach an omnidirectional and broadband enhancement in both optical absorption and quantum efficiency. The photocurrent density up to $3.5 \mathrm{~mA} \mathrm{~cm} \mathrm{~cm}^{-2}$ was obtained with only $120 \mathrm{~nm}$ thick CdS film on PTP (height $=270 \mathrm{~nm}$, aspect ratio of $\mathrm{P} / \mathrm{TP}=0.5$ ). Moreover, the activity of the as-prepared photoanode is almost angle independent and only less than $8 \%$ loss was observed from 0 to 40 degree.

\section{Results and discussion}

The process for fabricating CdS/Au PTP structures is revealed in Figure 1a. The details can be found in the experimental section. Briefly, a first-step anodization was carried out on an imprinted aluminum foil to grow anodic aluminum oxide (AAO) template. By dissolving the AAO template in $\mathrm{H}_{3} \mathrm{PO}_{4}$ solution, aluminum nanotips were observed at the fourfold junction sites of anodized nanopores. Then, a second-step anodization was conducted to form inverted PTP nanopores. An Au (100 nm)/Ti (5 nm) layer was deposited on the surface of the inverted PTP nanopores followed by growth of a thick Ni supporting film. An array of Au PTP was achieved by removing the unoxidized aluminum foil and AAO template, as the SEM images shown in Figure $1 \mathrm{~b}$ and 1c. The profile of the PTP structure includes a P (height: $180 \mathrm{~nm}$, size: $150 \mathrm{~nm}$ ) on the top and a TP (height: $180 \mathrm{~nm}$, size of the front- and back-side square surfaces: 
150 and $370 \mathrm{~nm}$ ) at the bottom. The $\mathrm{P}$ height can be easily tuned by altering the second-step anodization time. Finally, a continuous $\mathrm{CdS}$ thin layer $(90 \mathrm{~nm})$ was uniformly coated on the $\mathrm{Au}$ PTP using an electrodeposition process, as the top-down and cross-sectional SEM images shown in Figure 1d and 1e.

The composition of the as-prepared CdS/Au PTPs was confirmed by XRD measurement (Figure 1f). Besides the diffraction peaks of Au (JCPDS No. 65-8601), the other peaks can be indexed to (002), (101), (102), (103), (112), (004) and (104) diffractions of hexagonal CdS (PDF\#65-3414). Meanwhile, an Au pillar array (with the same height of Au PTPs, Figure S1) and an Au planar coated with a $90 \mathrm{~nm}$ CdS film were used as the comparison samples. The absorption spectra of the CdS/Au PTPs, along with the CdS/Au pillars and planar, were collected under a diffuse reflectance mode (Figure 1g). The CdS/Au planar presents an absorption onset at $520 \mathrm{~nm}$, in consistence with the bandgap of bulk CdS $(2.42 \mathrm{eV}) .{ }^{42}$ More importantly, the CdS/Au PTPs shows a superior optical absorption of about $95 \%$ within the wavelength range of $300-600 \mathrm{~nm}$. And a better optical absorption above $500 \mathrm{~nm}$ was also observed for the CdS/Au PTPs in contrast to the CdS/Au pillar, which agrees with the tendency of the Au PTPs and pillars absorption, indicating that the optical enhancement should be ascribed to the distinctive structure of the $\mathrm{Au}$ PTPs (Figure S2). 

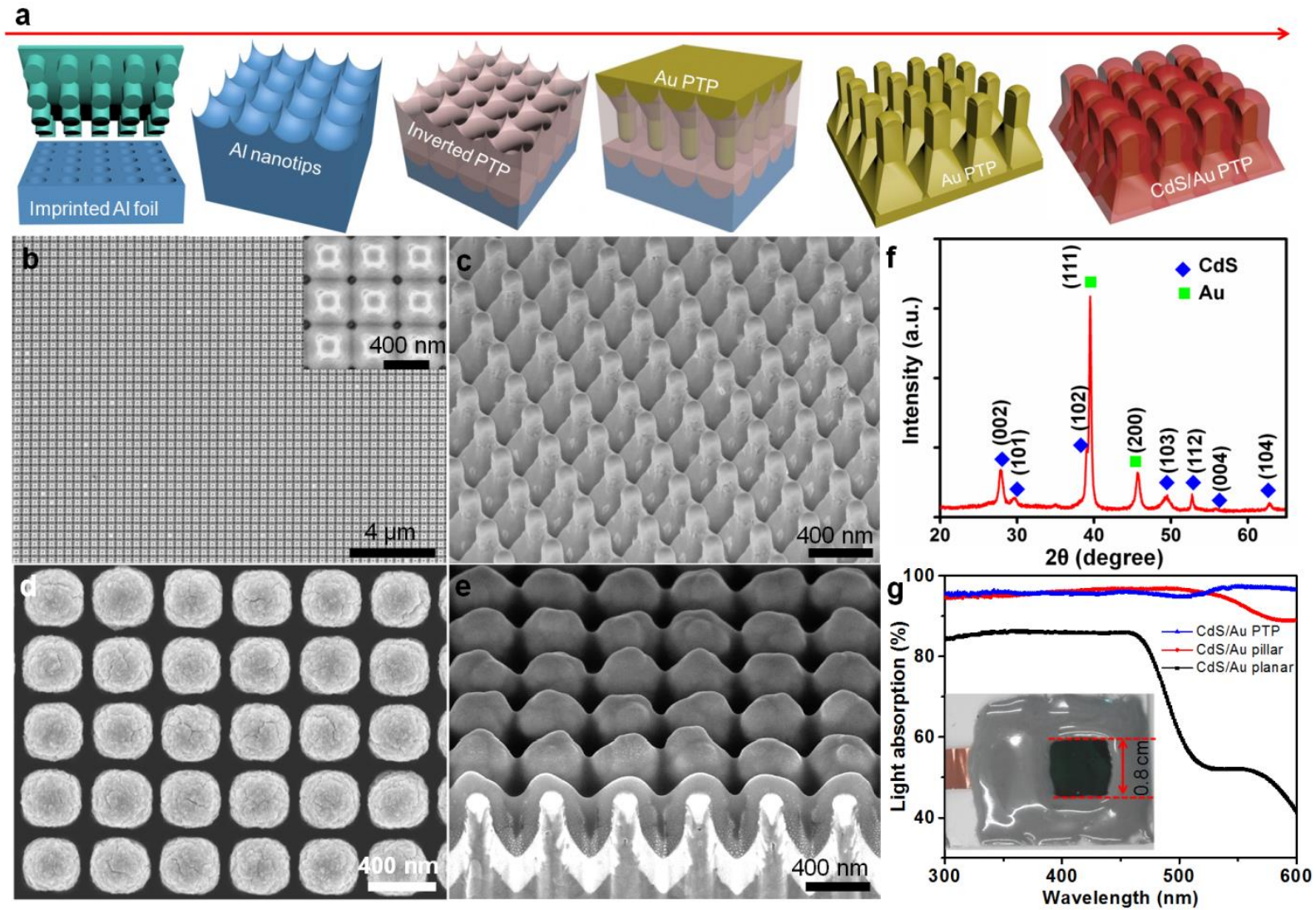

Figure 1. (a) Schematic of the fabrication process for CdS/Au PTP nanostructure array. (b) Large-area and (c) tilted SEM view of an Au PTP array, including top pillar (height: $180 \mathrm{~nm}$, size: $150 \mathrm{~nm}$ ) and bottom truncated-pyramid (height: $180 \mathrm{~nm}$, size of the front- and back-side square surfaces: 150 and $370 \mathrm{~nm}$, respectively). (d) Top-down and (e) cross-sectional SEM view of a CdS/Au PTP array coated with a $90 \mathrm{~nm}$ CdS film. (f) XRD of the as-prepared CdS/Au PTPs. (g) Light absorption spectra of the $\mathrm{CdS} / \mathrm{Au}$ PTPs, CdS/Au pillars, and $\mathrm{CdS} / \mathrm{Au}$ planar, respectively. Inset: photo of a typical CdS/Au PTPs.

The PEC performance was measured in a mixed aqueous solution $(0.2 \mathrm{M} \mathrm{Na} 2 \mathrm{~S}$ and $1.0 \mathrm{M}$ $\mathrm{Na}_{2} \mathrm{~S}_{2} \mathrm{O}_{3}$ ) using a three-electrode cell under the illumination of AM 1.5G. The chopped I-V curves were collected in Figure 2a. The photocurrent density was significantly enhanced for the 
CdS/Au PTPs when compared to that of the CdS/Au pillars and planar, with an enhancement of about $40 \%$ and $125 \%$ at a bias of $-0.4 \mathrm{~V}$ (vs. $\mathrm{Ag} / \mathrm{AgCl}$ ), respectively. Moreover, because the onset potential of the photocurrent remained the same for all three photoanodes, it can be concluded that the underneath Au has not been involved in a surface catalysis process at the semiconductor-electrolyte interface. The durability of the CdS/Au PTPs was tested at a bias of $0.4 \mathrm{~V}$ (vs. $\mathrm{Ag} / \mathrm{AgCl}$ ), in which the photocurrent maintained almost no degradation under AM 1.5G illumination over 100 minutes (Figure S3). Also, the CdS/Au PTPs photoanode exhibits excellent thermal robustness after an annealing treatment at $350{ }^{\circ} \mathrm{C}$, because the photocurrent even shows a slight improvement. The reason may be ascribed to the crystallization of the CdS film, which can be evidenced by the emergence of grains on the CdS surface and the intensity enhancement of the CdS diffraction peaks (Figure S4). On the other hand, spectrally resolved IPCE was measured in the same solution using a two-electrode cell without applying external bias. The CdS/Au PTPs showed a substantially IPCE enhancement within the wavelength range of $300-600 \mathrm{~nm}$ when compared with that of the $\mathrm{CdS} / \mathrm{Au}$ planar, agreeing with the tendency of the photocurrent results (Figure 2a). In the meantime, a better IPCE enhancement over $450 \mathrm{~nm}$ was also observed for the CdS/Au PTPs than that of the CdS/Au pillars (Figure 2b). The difference is more clearly from the normalized IPCE in Figure 2c, where a wavelength dependent IPCE enhancement with two peaks at 490 and $550 \mathrm{~nm}$ was observed for the $\mathrm{CdS} / \mathrm{Au}$ PTPs, while only one peak at $490 \mathrm{~nm}$ for the CdS/Au pillars.

Since the light absorption in the semiconductor $(\mathrm{CdS})$ and in the metallic $(\mathrm{Au})$ regions is hard to distinguish, theoretical FDTD simulations were employed to elucidate the optical difference between the CdS/Au PTPs and pillars. When SPR is excited, the collective charge oscillations at the metal surface cause increased the ohmic loss of EM energy, which can be visualized as peaks 
in the spectrally resolved absorption of the metal ${ }^{43}$ By extracting the absorption in the Au region, the EM energy dissipated at the top P $(180 \mathrm{~nm})$ and bottom TP $(180 \mathrm{~nm})$ of Au PTPs were obtained, respectively (Figure 2d). It shows that the top P part has two distinct absorption peaks at 500 and $550 \mathrm{~nm}$, while the bottom TP part has one major peak at $525 \mathrm{~nm}$ and one shoulder at $550 \mathrm{~nm}$. These Au absorption peaks are generally coincident with the normalized IPCE features of the CdS/Au PTP (Figure 2c). Small peak shift might originate from a discrepancy between the experiment and simulation, like the variation of the PTP geometry, the surface roughness of $\mathrm{CdS} / \mathrm{Au}$ PTPs that is unavoidable in reality but not considered in the simulation. For a comparison, the FDTD simulation concerning the Au pillar was also studied. The top part of Au pillars (top P: $180 \mathrm{~nm}$ ) shows two absorption peaks at 500 and $525 \mathrm{~nm}$, which is quite similar to that of the Au PTPs (top P: $180 \mathrm{~nm}$ ). However, no absorption peak from the bottom part of the Au pillars (bottom P: $180 \mathrm{~nm}$ ) was observed, which explains the loss of the normalized IPCE peak at $550 \mathrm{~nm}$ for $\mathrm{CdS} / \mathrm{Au}$ pillars. These results can be further supported by the electric field distribution on CdS/Au PTPs and pillars at $550 \mathrm{~nm}$ (Figure 2e). The red and yellow color areas in CdS present relatively strong electric fields, which uniformly distribute around the whole profile of the PTP, but only partially focus on the top part of the pillar. Therefore, the IPCE enhancement over $450 \mathrm{~nm}$ should be mainly caused by the SPR modes that generate at the CdS$\mathrm{Au}$ interface and result in an enhanced light absorption in CdS layer. This point can be further confirmed by a CdS photoande based on weakly- or non- plasmonic metals (e.g., Pt) PTP structure, where the IPCE enhancement can be barely observed in the long wavelength range (Figure S5). 

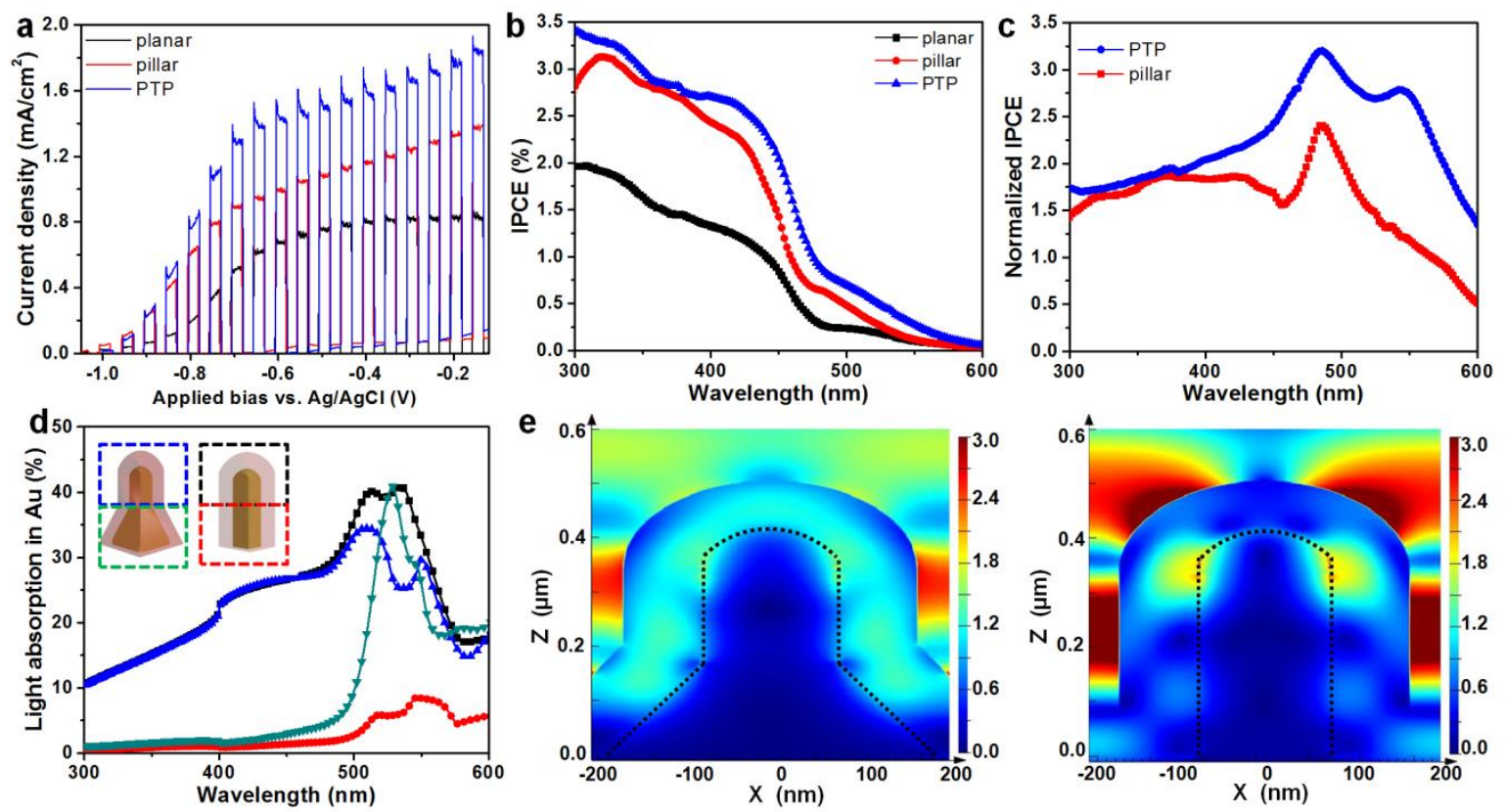

Figure 2. (a) Chopped photocurrent densities of CdS/Au PTPs, pillars, and plane under AM 1.5G illumination. (b) Spectrally resolved IPCE curves of the CdS/Au PTPs, pillars and plane within the wavelength range of $300-600 \mathrm{~nm}$ without applying an extra bias. (c) Normalized IPCE curves of the CdS/Au PTPs and pillars, which were calculated by dividing the IPCE of $\mathrm{CdS} / \mathrm{Au}$ PTPs and pillars by that of the CdS/Au plane, respectively. (d) FDTD simulated absorption curves of the top $(180 \mathrm{~nm})$ and bottom $(180 \mathrm{~nm})$ parts of the Au PTPs and pillars in CdS/Au PTPs and pillars, respectively. (e) FDTD simulated electric field distributions $\left|\mathrm{E}^{\mathrm{E}} / \mathrm{E}_{0}\right|$ of the CdS/Au PTPs (left) and pillars (right) at the wavelength of $550 \mathrm{~nm}$.

On the other hand, because no SPR peak was observed below $450 \mathrm{~nm}$, the IPCE enhancement within the range of $300-450 \mathrm{~nm}$ might be ascribed to the photonic modes and/or the increased surface area of the nanostructure. Considering that, CdS/Au PTPs with different P/TP height ratios $(0.1,0.5,1.0)$ were prepared, in which the surface area increases gradually (Figure 3a). The chopped I-V curves of these photoanodes revealed that the photocurrent reaches to a 
maximal value of about $2.5 \mathrm{~mA} \mathrm{~cm}^{-2}$ at a bias of $-0.4 \mathrm{~V}(\mathrm{vs} . \mathrm{Ag} / \mathrm{AgCl}$ ) when the ratio of $\mathrm{P} / \mathrm{TP}$ is 0.5 , instead of increasing with the raise of the photoanode surface area (Figure 3b). Moreover, from the normalized IPCE of CdS/Au PTPs in Figure 3c, the P/TP (0.5) shows an even IPCE enhancement across the whole wavelength range with a magnitude of about $300 \%$ and $150 \%$ to that of the P/TP (0.1) and P/TP (1.0), respectively. Thus, the change of surface area has an ignorable effect, while the photonic modes resulted strong light scattering at the interface of the $\mathrm{CdS}$ and Au plays a dominant role in the enhanced photocurrent below $450 \mathrm{~nm}$. These results can also be supported by the FDTD simulation results (Figure 3c). Comparing to the PTP $(0.1)$ and (1.0), the PTP (0.5) demonstrates a strong electric field distribution not only at the top and side wall but also in the valley between the neighboring PTPs. More importantly, we can conclude that with an appropriate aspect ratio of $\mathrm{P} / \mathrm{TP}$, the SPR and photonic modes can efficiently compensate each other to improve the light utilization in CdS layer over a wide range of wavelengths. 

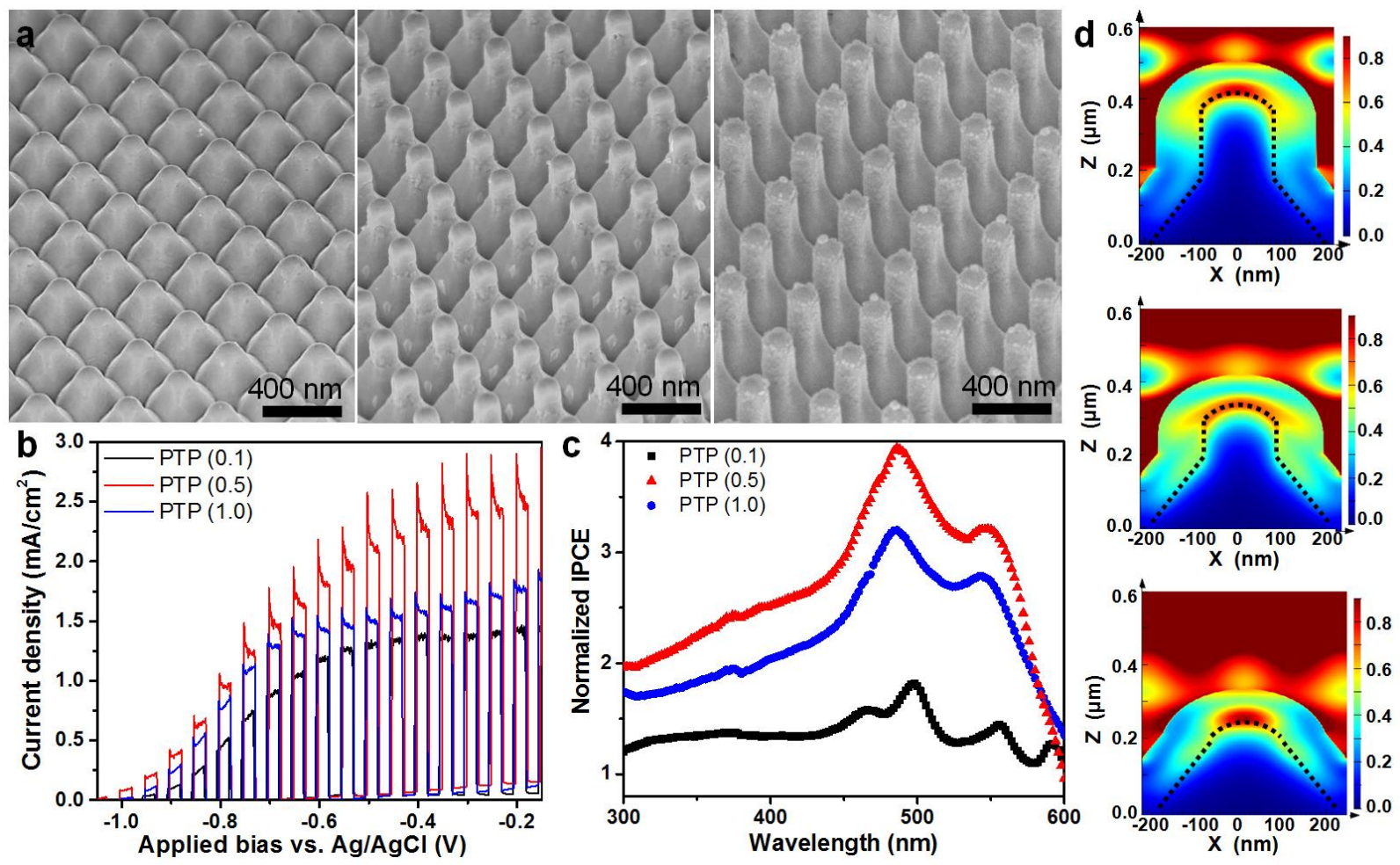

Figure 3. (a) SEM view of typical CdS/Au PTPs with different P/TP height ratios. From left to right: 0.1, 0.5, and 1.0, respectively. (b) Chopped photocurrent densities of the CdS/Au PTPs under AM 1.5G illumination. (c) Normalized IPCE curves of the CdS/Au PTPs, which were calculated by dividing the IPCE of the CdS/Au PTPs by that of the CdS/Au plane. (d) FDTD simulated electric field distributions $\left|\mathrm{E} / \mathrm{E}_{0}\right|$ of the CdS/Au PTPs with different P/TP ratios at the wavelength of $350 \mathrm{~nm}$. From top to bottom: 1, 0.5, and 0.1, respectively.

Besides the metallic nanostructure, SPR effect also depends upon the dielectric environment surrounding the metal. ${ }^{44,45}$ Because of the subwavelength thickness of the CdS layer, the effective refractive index is an average of the CdS layer and the electrolyte. ${ }^{40,46,47}$ Thus, the wavelength related photocurrent is possible to be tuned by changing CdS thickness. With deposition time elongation, the architecture of CdS/Au PTPs (0.5) gradually evolved from a 
core/shell structure to a planar film and the uniformity of the deposited CdS layers over large scales can be confirmed in Figure 4d and Figure S6. The chopped I-V curves reveal that the photocurrent density was enhanced more than two times with the increase of CdS thickness from 60 to $120 \mathrm{~nm}$ (Figure 4a). When the CdS thickness was further increased, a merged CdS film was formed with a pronounced decline of photocurrent. Because the normalized IPCE peaks in the long wavelength region show minimal wavelength shift, SPR effects play a similar role in all samples (Figure $4 \mathrm{~b}$ ). But the absolute values of the normalized IPCE alter significantly with the change of CdS thickness, implying that the photonic modes strongly depend on the $\mathrm{CdS}$ thickness and the formed nanostructure profile. The FDTD simulated electric field distribution of the CdS/Au PTPs are in accordance with the IPCE results. Obviously, the coverage of the strong electric field in the CdS layer becomes broader with the CdS thickness increasing from 60 to 120 nm. After the CdS layer merged to a planar film, surface-profile-induced photonic modes vanished, which results in a sharp shrink of the strong electric field in the CdS layer (Figure 4c).
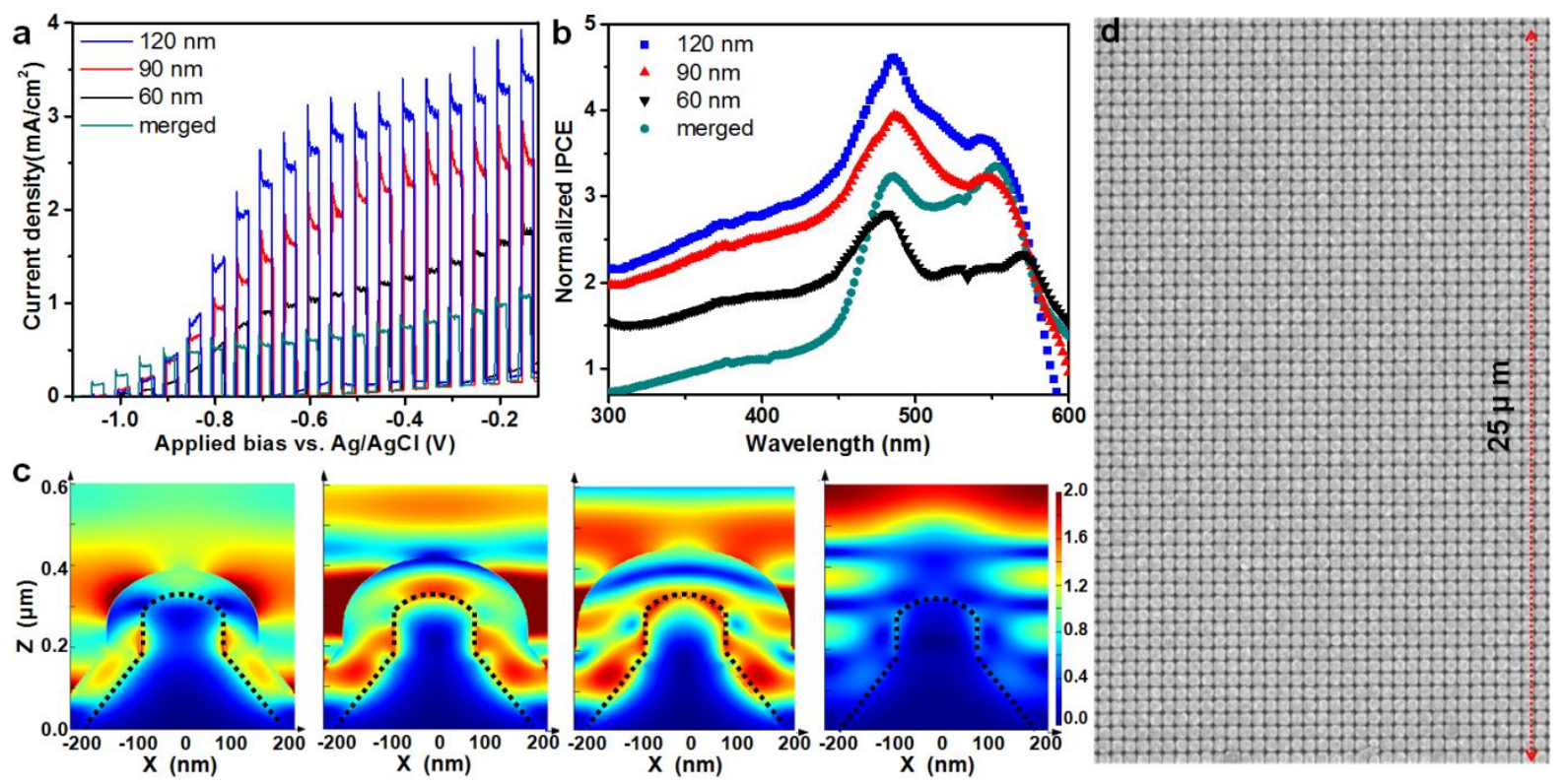
Figure 4. (a) Chopped photocurrent densities of the CdS/Au PTPs (0.5) with different CdS thicknesses under AM 1.5G illumination. (b) Normalized IPCE curves of the CdS/Au PTPs (0.5) with different CdS thicknesses, which are calculated by dividing the IPCE of the CdS/Au PTPs (0.5) by that of the $\mathrm{CdS} / \mathrm{Au}$ planar, respectively. (c) FDTD simulated electric field distributions $\left|\mathrm{E} / \mathrm{E}_{0}\right|$ of the CdS/Au PTPs (0.5) with different CdS thicknesses at the wavelength of $550 \mathrm{~nm}$. From left to right: $60 \mathrm{~nm}, 90 \mathrm{~nm}, 120 \mathrm{~nm}$, and merged, respectively. (d) Top-down SEM image of a typical large-scale CdS/Au PTP (0.5).

It should be noted that all previous measurements were performed under the normal incidence of AM 1.5G illumination and from a practical perspective, the investigation of incident angle effect is essential. The wavelength-resolved optical absorption of the CdS/Au PTPs (0.5) as a function of incident angle was calculated by FDTD simulations. The results revealed that only a slight variation is observed in the photonic-modes dominated short wavelength region, while the optical loss is mainly generated over long wavelength region that is dominated by the SPR modes (Figure 5a). In order to evaluate the overall light absorption of the CdS/Au PTPs, the averaged light absorption (ALA) is employed, which is calculated by dividing the solar photons absorbed in the CdS/Au PTPs by the total solar photons in the wavelength range of 300-600 nm [Equation 1]..$^{48,49}$

$$
A L A=\frac{\int_{\lambda 0}^{\lambda 1} L A(\lambda) * \frac{\lambda}{h c} * I_{A M 1.5}(\lambda) d \lambda}{\int_{\lambda 0}^{\lambda 1} \frac{\lambda}{h c} * I_{A M 1.5}(\lambda) d \lambda}
$$


where $\mathrm{h}$ is Planck's constant, $\mathrm{c}$ is light speed in free space, $\lambda$ is light wavelength ( $\lambda 0$ and $\lambda 1$ equal 300 and $600 \mathrm{~nm}$, respectively) and $\mathrm{I}_{\mathrm{AM} 1.5}$ is the solar spectrum of $\mathrm{AM} 1.5 \mathrm{G}$, LA is the simulated light absorption in Figure 5a. All ALA maintain around 90\% regardless of the incident angle which implies an almost angle-independent PEC performance (Figure 5b). Note that, a slight reduction of the photocurrent density $(<8 \%)$ was observed as the incident angle is altered from 0 to 40 degree (Figure $5 \mathrm{~b}$ and $\mathrm{S} 7$ ). It can be ascribed to the nonuniform electric field distributions that increasing incident angle results in spatial shrinkage of the strong electric filed in the CdS layer, which will lead to stronger recombination of the photogenerated carriers and consequently, a somewhat deteriorated photocurrent (Figure S8).
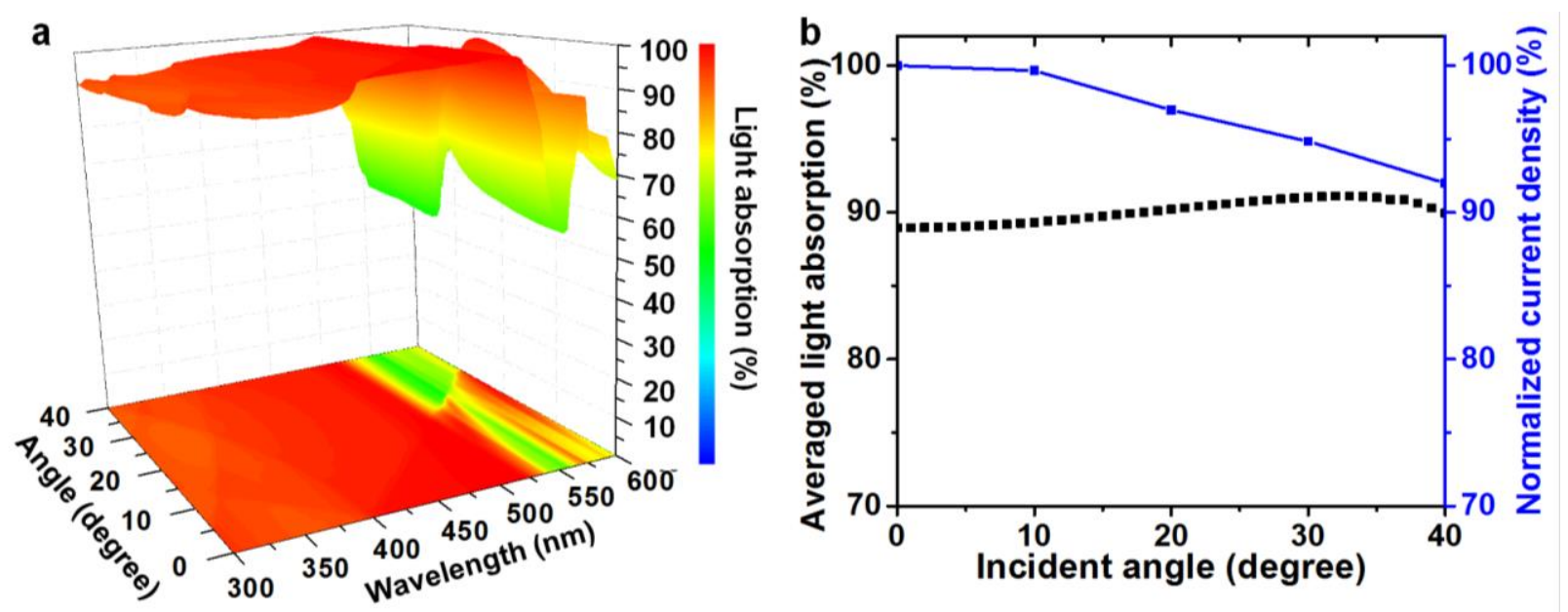

Figure 5. Characterization of the CdS/Au PTPs (0.5) with $120 \mathrm{~nm} \mathrm{CdS} \mathrm{as} \mathrm{a} \mathrm{function} \mathrm{of} \mathrm{incident}$ angle. (a) Simulated spectrally-resolved light absorption. (b) Averaged light absorption and normalized photocurrent density under $\mathrm{AM} 1.5 \mathrm{G}$ illumination at $-0.4 \mathrm{~V}$ vs. $\mathrm{Ag} / \mathrm{AgCl}$.

\section{Conclusions}


Perfectly ordered arrays of CdS/Au PTP nanostructures were successfully fabricated with a combination of nanoengineered AAO template and conventional techniques (e.g., PVD and electrodeposition). A significant IPCE enhancement was observed by taking advantage of SPR and photonic modes root in Au PTPs, where the SPR modes dominate in the longer wavelength range over $450 \mathrm{~nm}$ and photonic modes contribute mainly in the shorter wavelength range below $450 \mathrm{~nm}$. Different Au PTP profile and CdS thickness were systematically investigated with experiments and FDTD simulations to reach an omnidirectional and broadband enhancement in both optical absorption and quantum efficiency. The photocurrent up to $3.5 \mathrm{~mA} \mathrm{~cm}{ }^{-2}$ was obtained from the CdS/Au PTP (0.5) with only less than $8 \%$ loss from 0 to 40 degree irradiation, four times of that from the planer $\mathrm{CdS} / \mathrm{Au}$. Moreover, the scalable strategy demonstrated here should not be limited to the CdS/Au PTP. Other plasmonic PTP, such as Ag, Al, Cu and alloyed metals together with thin semiconductor materials are highly expectable for cost-effective photovoltaic and photochemical electrodes.

\section{Experimental Section}

Fabrication of CdS/Au PTPs photoanodes. 1) A Ni imprinting mould with periodic pillar arrays of $400 \mathrm{~nm}$ pitch was prepared using a Si mother mould (AMO GmbH). Polished Al foils were imprinted using the as-prepared Ni mould to obtain a periodic-nanodent pattern. 2) The first-step anodization was performed on the imprinted $\mathrm{Al}$ foils in a $5 \mathrm{wt} \%$ phosphoric acid under a constant voltage of $160 \mathrm{~V}$ for 5 minutes which was followed by the AAO dissolution into an aqueous solution ( $1.5 \mathrm{wt} \%$ chromium acid and $6 \mathrm{wt} \%$ phosphoric acid) at $\left.60^{\circ} \mathrm{C} .3\right)$ The secondstep anodization was carried out in the same condition as outlined in the first-step anodization. And elongating anodization time led to the height increase of the bottom hole of the inverted 
PTP AAO template. 4) The inverted PTP AAO template was coated with a $5 \mathrm{~nm}$ Ti and $100 \mathrm{~nm}$ Au layer using PVD followed by the electrochemical deposition of a thick Ni film under a constant current of $5.0 \mathrm{~mA} / \mathrm{cm}^{2}$ in an aqueous electrolyte $\left(0.38 \mathrm{M} \mathrm{NiSO}_{4}, 0.12 \mathrm{M} \mathrm{NiCl}_{2}\right.$, and 0.5 $\mathrm{M} \mathrm{H}_{3} \mathrm{BO}_{3}$ ). 5) An Au PTP array was attained after etching the remainng $\mathrm{Al}$ in a mixed aqueous solution (27 $\mathrm{wt} \% \mathrm{CuCl}_{2}$ and $\left.3 \mathrm{wt} \% \mathrm{HCl}\right)$ and $\mathrm{AAO}$ template in $5 \mathrm{wt} \%$ phosphoric acid. 6) $\mathrm{CdS}$ was cathodically electrodeposited under a constant current of $1.0 \mathrm{~mA} / \mathrm{cm}^{2}$ at $130^{\circ} \mathrm{C}$ in a twoelectrode electrochemical bath where a Pt mesh and the as-fabricated Au PTPs served as the counter electrode and working electrode, respectively. The electrolyte was made by dissolving $3.6 \mathrm{~g} \mathrm{CdCl}_{2}$ and $1.6 \mathrm{~g} \mathrm{~S}$ in dimethyl sulfoxide (DMSO). Thermal treatment of the sample was carried out in ambience at $350^{\circ} \mathrm{C}$ for 30 minutes with $5^{\circ} \mathrm{C} / \mathrm{min}$ heating rate and then naturally cooled to room temperature.

Photoelectrochemical performance measurement. Oriel solar simulator (300 W Xe lamp, AM 1.5 global filter) was taken as the simulated light source and calibrated to AM $1.5 \mathrm{G}$ by a standard Si photodiode (Model 818, Newport). The photocurrent density was measured using a threeelectrode electrochemical cell, in which $\mathrm{Na}_{2} \mathrm{~S} / \mathrm{Na}_{2} \mathrm{~S}_{2} \mathrm{O}_{3}(0.2 \mathrm{M} / 1 \mathrm{M})$ aqueous solution served as the electrolyte, the CdS/Au PTPs acted as the working electrode, the Pt mesh worked as the counter electrode, and an $\mathrm{Ag} / \mathrm{AgCl}$ electrode functioned as the reference electrode. IPCE measurements were performed using the same electrolyte in a two-electrode electrochemical cell under no external bias with QEPVSI-b Quantum Efficiency Measurement System (Newport).

Characterizations. SEM measurements and the cross-section cutting process were performed by Auriga Zeiss FIB. XRD tests were conducted using a Bruker-AXS Discover D8 applying the $\mathrm{Cu}$ 
$\mathrm{K} \alpha(1.54056 \AA)$ radiation equipment. Ultraviolet-visible absorbance spectroscopy was carried out with Varian Cary 5,000 UV-vis-NIR spectrophotometer.

Theoretical Calculations. All simulations were performed using FDTD simulation software package from Lumerical Computational solutions, Inc. 3D layout was employed for the theoretical simulation in which periodic boundary condition was applied along the $\mathrm{x}$ and $\mathrm{y}$ axes with the periodicities of $400 \mathrm{~nm}$, and the $\mathrm{z}$ axis was truncated by perfectly matched layers (PML) condition at $\mathrm{z}=-500 \mathrm{~nm}$ and $\mathrm{z}=2000 \mathrm{~nm}$. Accordingly, a unit cell of the investigated array was simulated. Simulated samples were set on a platform which was parallel with the xy plane and was $1000 \mathrm{~nm}$ below the light source. Plane-wave light source, which polarized in the $\mathrm{x}$ direction and propagated along $-\mathrm{z}$ direction, was exploited to illuminate the samples. All geometrical parameters of the sample were set according to the experimental measurements. The permittivity for Au was taken from Johnson and Christy, and the experimental data in Treharne's literature were used for the dielectric index for $\mathrm{CdS} .^{50}$ In all simulations, mesh accuracy was set to be 6 and mesh override region includes all $\mathrm{CdS} / \mathrm{Au}$ nanostructures in which the maximum mesh step is set to be $1 \mathrm{~nm}$. Electric field distributions were recorded through two-dimensional field profile monitors. The reflection was detected using a power monitor placed $500 \mathrm{~nm}$ up the light source. Because of the opaqueness of all samples, the transmission was set to be 0 . Light absorption in the Au nanostructures was calculated using Equation 2:

$$
P_{a b s}=-0.5 \omega|E|^{2} \operatorname{imag}(\varepsilon)
$$


in which $\omega=2 \pi / \lambda$ and $\lambda$ is the illumination wavelength, $E$ is the electric field intensity and $\varepsilon$ is the Au permittivity. The electric field intensity and Au permittivity were measured by a threedimensional frequency-domain field monitor and a refractive index monitor, respectively.

\section{ASSOCIATED COTENT}

Supporting Information. SEM images of Au pillars, optical absorption spectra of Au PTPs and pillars, phototability of CdS/Au PTPs, SEM images of CdS/Au PTPs with different thickness of CdS layer, photocurrent density and electric field distributions of CdS/Au PTPs under different incident angle illumination.

This Supporting Information is available free of charge via the Internet at http://pubs.acs.org.

\section{AUTHOR INFORMATION}

\section{Corresponding Author}

*E-mail: yong.lei@tu-ilmenau.de.

\section{Author Contributions}

${ }^{\dagger}$ R.X. and L.Y.W. contributed equally to this work.

\section{ACKNOWLEDGMENT}

This work is financially supported by the European Research Council (ThreeDsurface, 240144), European Research Council (HiNaPc, 737616), Federal Ministry of Education and Research in Germany (BMBF, ZIK-3DNanoDevice, 03Z1MN11), BMBF (Meta-ZIK-BioLithoMorphie, 03Z1M512), German Research Foundation (DFG: LE 2249_4-1), Beijing Natural Science Foundation (2162042) and National Natural Science Foundation of China (21503209). Prof. Z. 
Wang appreciates the support from Hundred-Talent Program (Chinese Academy of Sciences),

Beijing Natural Science Foundation (2162042) and the National Natural Science Foundation of China (21503209), Key Research Program of Frontier Science, CAS (Grant No. QYZDB-SSWSLH006).

\section{REFERENCES}

1. Lewis, N. S.; Nocera, D. G., Powering the Planet: Chemical Challenges in Solar Energy Utilization. Proc. Natl. Acad. Sci. U. S. A. 2006, 103, 15729-15735.

2. Kamat, P. V., Meeting the Clean Energy Demand: Nanostructure Architectures for Solar Energy Conversion. J. Phys. Chem. C 2007, 111, 2834-2860.

3. Thirugnanasambandam, M.; Iniyan, S.; Goic, R., A Review of Solar Thermal Technologies. Renewable Sustainable Energy Rev. 2010, 14, 312-322.

4. Green, M. A.; Emery, K.; Hishikawa, Y.; Warta, W.; Dunlop, E. D., Solar Cell Efficiency Tables (Version 45). Prog. Photovolt: Res. Appl. 2015, 23, 1-9.

5. Montoya, J. H.; Seitz, L. C.; Chakthranont, P.; Vojvodic, A.; Jaramillo, T. F.; Norskov, J. K., Materials for Solar Fuels and Chemicals. Nat. Mater. 2016, 16, 70-81.

6. Fujishima, A.; Honda, K., Electrochemical Photolysis of Water at a Semiconductor Electrode. Nature 1972, 238, 37-38.

7. Zou, Z.; Ye, J.; Sayama, K.; Arakawa, H., Direct Splitting of Water Under Visible Light Irradiation with an Oxide Semiconductor Photocatalyst. Nature 2001, 414, 625-627. 
8. Maeda, K.; Teramura, K.; Lu, D. L.; Takata, T.; Saito, N.; Inoue, Y.; Domen, K., Photocatalyst Releasing Hydrogen from Water - Enhancing Catalytic Performance Holds Promise for Hydrogen Production by Water Splitting in Sunlight. Nature 2006, 440, 295-295.

9. Zhang, Z. H.; Dua, R.; Zhang, L. B.; Zhu, H. B.; Zhang, H. N.; Wang, P., Carbon-LayerProtected Cuprous Oxide Nanowire Arrays for Efficient Water Reduction. Acs Nano 2013, 7, 1709-1717.

10. Wang, X.; Xie, J.; Li, C. M., Architecting Smart "Umbrella" $\mathrm{Bi}_{2} \mathrm{~S}_{3} / \mathrm{rGO}-\mathrm{Modified} \mathrm{TiO}_{2}$ Nanorod Array Structures at the Nanoscale for Efficient Photoelectrocatalysis under Visible Light. J. Mater. Chem. A 2015, 3, 1235-1242.

11. Mi, Y.; Wen, L.; Xu, R.; Wang, Z.; Cao, D.; Fang, Y.; Lei, Y., Constructing a AZO/TiO 2 Core/Shell Nanocone Array with Uniformly Dispersed $\mathrm{Au}$ NPs for Enhancing Photoelectrochemical Water Splitting. Adv. Energy Mater. 2016, 6, 1501496.

12. Liu, Y.; Liang, L.; Xiao, C.; Hua, X.; Li, Z.; Pan, B.; Xie, Y., Promoting Photogenerated Holes Utilization in Pore-Rich $\mathrm{WO}_{3}$ Ultrathin Nanosheets for Efficient Oxygen-Evolving Photoanode. Adv. Energy Mater. 2016, 6, 1600437.

13. Lei, F.; Zhang, L.; Sun, Y.; Liang, L.; Liu, K.; Xu, J.; Zhang, Q.; Pan, B.; Luo, Y.; Xie, Y., Atomic-Layer-Confined Doping for Atomic-Level Insights into Visible-Light Water Splitting. Angew. Chem., Int. Ed. 2015, 54, 9266-9270. 
14. Xie, J.; Guo, C.; Yang, P.; Wang, X.; Liu, D.; Li, C. M., Bi-Functional Ferroelectric $\mathrm{BiFeO}_{3}$ Passivated $\mathrm{BiVO}_{4}$ Photoanode for Efficient and Stable Solar Water Oxidation. Nano Energy 2017, 31, 28-36.

15. Hou, W. B.; Cronin, S. B., A Review of Surface Plasmon Resonance-Enhanced Photocatalysis. Adv. Funct. Mater. 2013, 23, 1612-1619.

16. Li, Y.; Takata, T.; Cha, D.; Takanabe, K.; Minegishi, T.; Kubota, J.; Domen, K., Vertically Aligned $\mathrm{Ta}_{3} \mathrm{~N}_{5}$ Nanorod Arrays for Solar-Driven Photoelectrochemical Water Splitting. Adv. Mater. 2013, 25, 125-131.

17. Maier, S. A., Plasmonics: Fundamentals and Applications. 1st ed.; Springer: Berlin, 2007.

18. Linic, S.; Christopher, P.; Ingram, D. B., Plasmonic-Metal Nanostructures for Efficient Conversion of Solar to Chemical Energy. Nat. Mater. 2011, 10, 911-921.

19. Guo, L. H.; Ferhan, A. R.; Chen, H. L.; Li, C. M.; Chen, G. N.; Hong, S.; Kim, D. H., Distance-Mediated Plasmonic Dimers for Reusable Colorimetric Switches: A Measurable Peak Shift of More than $60 \mathrm{~nm}$. Small 2013, 9, 234-240.

20. Kim, H. J.; Lee, S. H.; Upadhye, A. A.; Ro, I.; Tejedor-Tejedor, M. I.; Anderson, M. A.; Kim, W. B.; Huber, G. W., Plasmon-Enhanced Photoelectrochemical Water Splitting with SizeControllable Gold Nanodot Arrays. ACS nano 2014, 8, 10756-10765.

21. Zhan, Z. B.; Grote, F.; Wang, Z. J.; Xu, R.; Lei, Y., Degenerating Plasmonic Modes to Enhance the Performance of Surface Plasmon Resonance for Application in Solar Energy Conversion. Adv. Energy Mater. 2015, 5, 1501654. 
22. Li, Z. B.; Meng, G. W.; Huang, Q.; Hu, X. Y.; He, X.; Tang, H. B.; Wang, Z. W.; Li, F. D., Ag Nanoparticle-Grafted PAN-Nanohump Array Films with 3D High-Density Hot Spots as Flexible and Reliable SERS Substrates. Small 2015, 11, 5452-5459.

23. Wang, Z.; Cao, D.; Wen, L.; Xu, R.; Obergfell, M.; Mi, Y.; Zhan, Z.; Nasori, N.; Demsar, J.; Lei, Y., Manipulation of Charge Transfer and Transport in Plasmonic-Ferroelectric Hybrids for Photoelectrochemical Applications. Nat. Commun. 2016, 7, 10348.

24. Kakavelakis, G.; Vangelidis, I.; Heuer-Jungemann, A.; Kanaras, A. G.; Lidorikis, E.; Stratakis, E.; Kymakis, E., Plasmonic Backscattering Effect in High-Efficient Organic Photovoltaic Devices. Adv. Energy Mater. 2016, 6, 1501640.

25. Atwater, H. A.; Polman, A., Plasmonics for Improved Photovoltaic Devices. Nat. Mater. 2010, $9,205-213$.

26. Chen, X.; Jia, B. H.; Saha, J. K.; Cai, B. Y.; Stokes, N.; Qiao, Q.; Wang, Y. Q.; Shi, Z. R.; Gu, M., Broadband Enhancement in Thin-Film Amorphous Silicon Solar Cells Enabled by Nucleated Silver Nanoparticles. Nano Lett. 2012, 12, 2187-2192.

27. Solarska, R.; Bienkowski, K.; Zoladek, S.; Majcher, A.; Stefaniuk, T.; Kulesza, P. J.; Augustynski, J., Enhanced Water Splitting at Thin Film Tungsten Trioxide Photoanodes Bearing Plasmonic Gold-Polyoxometalate Particles. Angew. Chem., Int. Ed. 2014, 126, 14420-14424.

28. Archana, P. S.; Pachauri, N.; Shan, Z. C.; Pan, S. L.; Gupta, A., Plasmonic Enhancement of Photoactivity by Gold Nanoparticles Embedded in Hematite Films. J. Phys. Chem. C 2015, $119,15506-15516$. 
29. Wang, X. T.; Liow, C.; Qi, D. P.; Zhu, B. W.; Leow, W. R.; Wang, H.; Xue, C.; Chen, X. D.; Li, S. Z., Programmable Photo-Electrochemical Hydrogen Evolution Based on MultiSegmented CdS-Au Nanorod Arrays. Adv. Mater. 2014, 26, 3506-3512.

30. Jeong, S.; McGehee, M. D.; Cui, Y., All-Back-Contact Ultra-Thin Silicon Nanocone Solar Cells with 13.7\% Power Conversion Efficiency. Nat. Commun. 2013, 4, 2950.

31. Kargar, A.; Sun, K.; Jing, Y.; Choi, C.; Jeong, H.; Jung, G. Y.; Jin, S.; Wang, D., 3D Branched Nanowire Photoelectrochemical Electrodes for Efficient Solar Water Splitting. ACS nano 2013, 7, 9407-9415.

32. Li, G. J.; Li, H.; Ho, J. Y. L.; Wong, M.; Kwok, H. S., Nanopyramid Structure for Ultrathin c-Si Tandem Solar Cells. Nano Lett. 2014, 14, 2563-2568.

33. Li, J.; Qiu, Y.; Wei, Z.; Lin, Q.; Zhang, Q.; Yan, K.; Chen, H.; Xiao, S.; Fan, Z.; Yang, S., A Three-Dimensional Hexagonal Fluorine-Doped Tin Oxide Nanocone Array: A Superior Light Harvesting Electrode for High Performance Photoelectrochemical Water splitting. Energy Environ. Sci. 2014, 7, 3651-3658.

34. Le Xie, J.; Guo, C. X.; Li, C. M., Construction of One-Dimensional Nanostructures on Graphene for Efficient Energy Conversion and Storage. Energy Environ. Sci. 2014, 7, 2559-2579.

35. Wen, L.; Wang, Z.; Mi, Y.; Xu, R.; Yu, S. H.; Lei, Y., Designing Heterogeneous 1D Nanostructure Arrays Based on AAO Templates for Energy Applications. Small 2015, 11, 34083428. 
36. Wang, Z.; Cao, D.; Xu, R.; Qu, S.; Wang, Z.; Lei, Y., Realizing Ordered Arrays of Nanostructures: A Versatile Platform for Converting and Storing Energy Efficiently. Nano Energy 2016, 19, 328-362.

37. Gao, H.; Liu, C.; Jeong, H. E.; Yang, P., Plasmon-Enhanced Photocatalytic Activity of Iron Oxide on Gold Nanopillars. ACS nano 2011, 6, 234-240.

38. Sherry, L. J.; Jin, R. C.; Mirkin, C. A.; Schatz, G. C.; Van Duyne, R. P., Localized Surface Plasmon Resonance Spectroscopy of Single Silver Triangular Nanoprisms. Nano Lett. 2006, 6, 2060-2065.

39. Mayer, K. M.; Lee, S.; Liao, H.; Rostro, B. C.; Fuentes, A.; Scully, P. T.; Nehl, C. L.; Hafner, J. H., A Label-Free Immunoassay Based Upon Localized Surface Plasmon Resonance of Gold Nanorods. Acs Nano 2008, 2, 687-692.

40. Xu, R.; Wang, X. D.; Song, L.; Liu, W.; Ji, A.; Yang, F. H.; Li, J. M., Influence of the Light Trapping Induced by Surface Plasmons and Antireflection Film in Crystalline Silicon Solar Cells. Opt. Express 2012, 20, 5061-5068.

41. Wen, L. Y.; Xu, R.; Mi, Y.; Lei, Y., Multiple Nanostructures Based On Anodized Aluminium Oxide Templates. Nat. Nanotechnol. 2017, 12, 244-250.

42. $\quad$ Li, H. Q.; Wang, X.; Xu, J. Q.; Zhang, Q.; Bando, Y.; Golberg, D.; Ma, Y.; Zhai, T. Y., One-Dimensional CdS Nanostructures: A Promising Candidate for Optoelectronics. Adv. Mater. 2013, 25, 3017-3037. 
43. Barnes, W. L.; Murray, W. A.; Dintinger, J.; Devaux, E.; Ebbesen, T. W., Surface Plasmon Polaritons and Their Role In the Enhanced Transmission of Light through Periodic Arrays of Subwavelength Holes in a Metal Film. Phys. Rev. Lett. 2004, 92, 107401.

44. Kelly, K. L.; Coronado, E.; Zhao, L. L.; Schatz, G. C., The Optical Properties of Metal Nanoparticles: the Influence of Size, Shape, and Dielectric Environment. J. Phys. Chem. B 2003, 107, 668-677.

45. Miller, M. M.; Lazarides, A. A., Sensitivity of Metal Nanoparticle Surface Plasmon Resonance to the Dielectric Environment. J. Phys. Chem. B 2005, 109, 21556-21565.

46. Damos, F. S.; Luz, R. C. S.; Kubota, L. T., Determination of Thickness, Dielectric Constant of Thiol Films, and Kinetics of Adsorption Using Surface Plasmon Resonance. Langmuir 2005, 21, 602-609.

47. Chien, F. C.; Chen, S. J., Direct Determination of the Refractive Index and Thickness of a Biolayer Based on Coupled Waveguide-Surface Plasmon Resonance Mode. Opt. Lett. 2006, 31, 187-189.

48. Zhang, Y. A.; Stokes, N.; Jia, B.; Fan, S. H.; Gu, M., Towards ultra-thin plasmonic silicon wafer solar cells with minimized efficiency loss. Sci. Rep. 2014, 4, 4939.

49. Yu, R.; Ching, K. L.; Lin, Q. F.; Leung, S. F.; Arcrossito, D.; Fan, Z. Y., Strong Light Absorption of Self-Organized 3-D Nanospike Arrays for Photovoltaic Applications. Acs Nano 2011, 5, 9291-9298. 
50. Treharne, R. E.; Seymour-Pierce A.; Durose K.; Hutchings K.; Roncallo S.; Lane D., Optical Design and Fabrication of Fully Sputtered CdTe/CdS Solar Cells. J. Phys.: Conf. Ser. 2011, 286, 012038. 